\title{
Large-scale synthesis of the carbon coils using stainless steel substrate
}

\author{
Young-Chul Jeon and Sung-Hoon $\mathrm{Kim}^{\dagger}$ \\ Department of Engineering in Energy \& Applied Chemistry, Silla University, Busan 617-736, Korea \\ (Received September 2, 2013) \\ (Revised November 8, 2013) \\ (Accepted November 15, 2013)
}

\begin{abstract}
Carbon coils could be synthesized using $\mathrm{C}_{2} \mathrm{H}_{2} / \mathrm{H}_{2}$ as source gases and $\mathrm{SF}_{6}$ as an incorporated additive gas under the thermal chemical vapor deposition system. A 304-type stainless steel was used as a substrate with nickel powders as the catalyst. The surface of the substrate was pretreated using a sand paper or a mechanical drill to enhance the production yield of the carbon coils. The characteristics of the deposited carbon nanomaterials on the substrates were investigated according to the surface state on the stainless steel substrate. The protrusion induced by the grooves on the substrate surface could enhance the formation of the carbon nanomaterials having the coils geometries. The cause for the enhancement of the carbon coils formation by the grooves was suggested and discussed with the surface energies for the interaction between as-growing carbon elements. Finally, we could obtain the massive production yield of the carbon coils by the surface pretreatment using $\mathrm{SiC}$ sand papers on the several tens grooved stainless steel substrate.
\end{abstract}

Key words Large-scale synthesis, Carbon coils, Stainless steel substrate, $\mathrm{SF}_{6}$ incorporation, Thermal chemical vapor deposition

\section{Introduction}

A particular interest in the synthesis of the carbon nanomaterials with a helical morphology, so called carbon coils, has risen dramatically in recent year. Because of an unique spring-like geometry and a chirality, the carbon coils have been regarded as the high potential materials for the catalyst and the electromagnetic wave absorbers, nano/micro-sized tactile sensors, actuators, resonators, mechanical springs, and so on [1-4].

The carbon coils have been occasionally found as the low-content byproducts of the vapor preparation of the carbon fibers in the microwave plasma-enhanced chemical vapor deposition or in the thermal chemical vapor deposition [5-8]. In addition, the carbon coils can be divided into the different categories based upon the helical nature of the material: single helix, double helix, triple helix, etc or the geometry size and shape: microcoil, nanocoil, wave-like nanocoil etc [9]. It was understood that the electrical properties of the one dimensional carbon materials like the carbon nanotubes were varied according to their geometries and sizes [10]. Up to the present, the control over the synthesis of a specific type of the helical carbon material has been met with only

\footnotetext{
Corresponding author

Tel: +82-51-999-5619

Fax: +82-51-999-5335

E-mail: shkim@silla.ac.kr
}

limited success. For the practical application of these materials, therefore, the controlled-characteristics for the coil morphology and geometry (diameter, pitch, length, and turning direction) with the large scale synthesis should be preferentially achieved.

Catalytic chemical vapor deposition (CCVD) has been found to be the reliable method that can produce the carbon coils [11]. However, due to the low yield of the carbon coils, normal CCVD process is not suitable for the scaled-up production of the carbon coils. To enhance the production yield of the carbon coils, various kinds of catalyst, substrate, and additives incorporation techniques have been widely investigated up to the present [12-23]. For the catalyst, Ni was known as an effective catalyst for the formation of the elastic carbon coils [12]. Co-silica or Fe-silica catalysts have been served as the substrates for the preparation of the carbon coils by catalytic pyrolysis of acetylene [13]. For the incorporated additives, meanwhile, a trace of the sulfur-related species was regarded as the promising additives for the formation of the carbon coils. The carbon coils were reported to be well-synthesized by adding a trace of hydrogen sulfide $\left(\mathrm{H}_{2} \mathrm{~S}\right)$ [14], carbon disulfide $\left(\mathrm{CS}_{2}\right)$ [15], thiophene $\left(\mathrm{C}_{4} \mathrm{H}_{4} \mathrm{~S}\right)$ as an impurity [16], or using $\mathrm{WS}_{2}$ as a catalytic material [17]. Previously, we also introduced the $\mathrm{SF}_{6}$ additive incorporation for the synthesis of the carbon nanofilaments [18-21]. $\mathrm{SF}_{6}$ was chosen to take the advantage for fluorine species characteristics regard- 
ing the enhancement of the nucleation sites for the carbon materials formation [22]. Furthermore, in-situ cycling on/ off modulation process for $\mathrm{C}_{2} \mathrm{H}_{2} / \mathrm{SF}_{6}$ flows was also introduced to explore a more effective and reliable CCVD method for the growth of the carbon coils [23].

Here, we introduced the attempt for the massive production of the carbon coils using a stainless steel substrate. Stainless steel is much easier to manufacture and, consequently, applicable to the massive production container. In addition, several kinds of stainless steel, such as type 304 , type 316 , and so on, have Ni element. So, the incorporated $\mathrm{Ni}$ element may play an assistant role for the essential catalyst for the formation of the carbon coils. Up to the present, merely a couple of works have been reported for the formation of the carbon coils using stainless steel substrate. Chang et al. introduced a simple method for growing carbon nanocoils on 304 stainless steels substrates [24]. The oxidation temperature and concentration of organic Sn solution were optimized for the high-yield growth of carbon nanocoils. Su et al. achieved the radial growth of carbon nanocoils on stainless steel wire using thermal chemical vapor deposition from acetylene [25]. Despite these valuable reports, the research on the enhancement of the carbon coils production yield using stainless steel substrate is still required to achieve the massive production yield of the carbon coils.

In this work we introduce a simple mechanical pretreatment method of the substrate to enhance the production yield of the carbon coils. Namely, the stainless steel substrates were pretreated by the scratching method using sand papers and/or the grooves formation using a mechanical drill. The characteristics (formation density, morphology, and geometry) of the deposited carbon coils were investigated and discussed according to the surface state on the stainless steel substrate.

\section{Experimental}

A 304 type stainless steel which has $1.5 \mathrm{~m}$ length, $10 \mathrm{~cm}$ width, and $1.0 \mathrm{~mm}$ thickness was used as the substrate. One side area of the substrate was pretreated
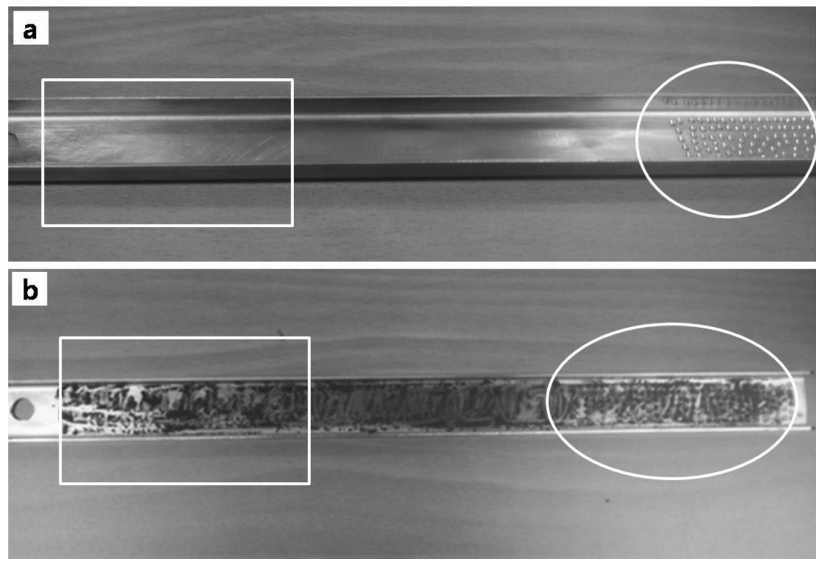

Fig. 1. Photographs of the stainless steel substrate pretreated by the unidirectional scratching using a $\mathrm{SiC}$ sand paper at one side area of the substrate (see the rectangular area) and by a mechanical drill (see the circular area) at the other side area of the substrate (see the circular area).

by the unidirectional scratching using a $\mathrm{SiC}$ sand paper as shown in the rectangular area in Fig. 1a. Several tens circular-shaped grooves were made on the other side area of the substrate using a mechanical drill (see the circular area in Fig. 1a). About $0.45 \mathrm{~g}$ Ni powders which have the diameters in the range of approximately $1 \sim 100 \mu \mathrm{m}$ were spread onto the substrate as shown in Fig. $1 b$.

For the carbon coils deposition reaction, thermal chemical vapor deposition (TCVD) system was employed. $\mathrm{C}_{2} \mathrm{H}_{2}$ and $\mathrm{H}_{2}$ were used as the source gases. $\mathrm{SF}_{6}$, an incorporated additive gas, was injected into the reaction chamber during the reaction. The flow rate for $\mathrm{C}_{2} \mathrm{H}_{2}, \mathrm{H}_{2}$, and $\mathrm{SF}_{6}$ were fixed at 15,35 , and 35 standard $\mathrm{cm}^{3}$ per minute $(\mathrm{sccm})$, respectively. The injection times for $\mathrm{C}_{2} \mathrm{H}_{2}$ and $\mathrm{H}_{2}$ flow were fixed at 120 minutes. However the additive gas $\left(\mathrm{SF}_{6}\right)$ flow was adjusted for 20 minutes from the starting point of the deposition reaction. The detailed reaction conditions were shown in Table 1 . The morphologies of carbon coils-deposited substrates were investigated using field emission scanning electron microscopy (FESEM, Hitach 4500). The qualities of the carbon coils according to the surface state of the substrate were investigated in the range of $800 \sim 2000 \mathrm{~cm}^{-1}$ by a microRaman spectrometer (Renishaw 2000) with about $50 \mu \mathrm{m}$ spot size of Ar-ion laser.

Table 1

Experimental conditions for the deposition of carbon coils on the substrate

\begin{tabular}{lllllllll}
\hline \hline $\begin{array}{l}\mathrm{C}_{2} \mathrm{H}_{2} \text { flow } \\
\text { rate }(\mathrm{sccm})\end{array}$ & $\begin{array}{l}\mathrm{H}_{2} \text { flow } \\
\text { rate }(\mathrm{sccm})\end{array}$ & $\begin{array}{l}\mathrm{SF}_{6} \text { flow } \\
\text { rate }(\mathrm{sccm})\end{array}$ & $\begin{array}{l}\text { Total pressure } \\
\text { (torr) }\end{array}$ & $\begin{array}{l}\text { Total deposition } \\
\text { time }(\mathrm{min})\end{array}$ & \multicolumn{3}{l}{ Source gases flow time (min) } & $\begin{array}{l}\text { Substrate } \\
\text { temp. }\left({ }^{\circ} \mathrm{C}\right)\end{array}$ \\
\hline 15 & 35 & 35 & 100 & 90 & $\mathrm{C}_{2} \mathrm{H}_{2}$ & $\mathrm{H}_{2}$ & $\mathrm{SF}_{6}$ & 120 \\
\hline
\end{tabular}




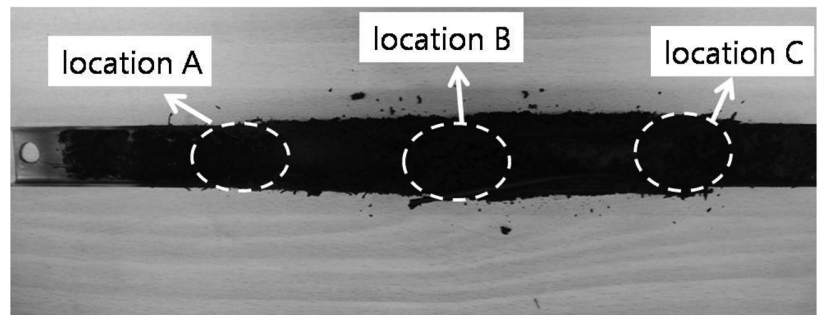

Fig. 2. Photograph of the as-grown carbon materials on the pretreated stainless steel substrate.

\section{Results and Discussion}

Fig. 2 shows the photographs of the as-grown carbon materials on the substrate. As a naked eye, a lot of the carbon materials were formed on the substrate. The measured amount of the deposited carbon materials was about $125 \mathrm{~g}$. As shown in Fig. 2, the center area of the substrate seems to produce a large amount of the carbon coils as compared with those of the side area of the substrate.

To investigate the effect of the pretreatment on the substrate, we measured the surface morphologies of the deposited carbon nanomaterials according to the locations on the substrate, namely the one side area pre- treated by the unidirectional scratching using a $\mathrm{SiC}$ sand paper (location $\mathrm{A}$ ), the center area without pretreatment (location B), and the other side area pretreated by several circular-shaped grooves using a mechanical drill (location C). Fig. 3 shows the microscopic images for the as-grown carbon materials according to the locations on the substrate. For location A, the formation of the linear type carbon nanofilaments was observed as shown in Figs. 3a d. Indeed, most of the linear type carbon nanofilaments have the board shapes. As shown in the arrow head of Fig. 3d, some of the board-shaped carbon nanofilaments show the wave-like geometries. For location B, any carbon nanofilament-like material couldn't be observed as shown in Figs. 3e h. Even in the high magnified (10k) FESEM images, merely the embryo-like morphologies could be observed (see Fig. $3 h)$. It reveals that the one-dimensional type carbon nanofilaments couldn't be developed on the substrate without the pretreatment. For location C, several-shaped carbon coils could be observed as shown in Figs. 3i 1. Indeed, a lot of carbon nanocoils (CNCs) having the various shapes could be observed on the substrate, compared with the carbon microcoils (CMCs). Particularly, the drill bit-type CNCs could be well observed (see Fig. 31). Estimating from Fig. 4, the lengths of CNCs in

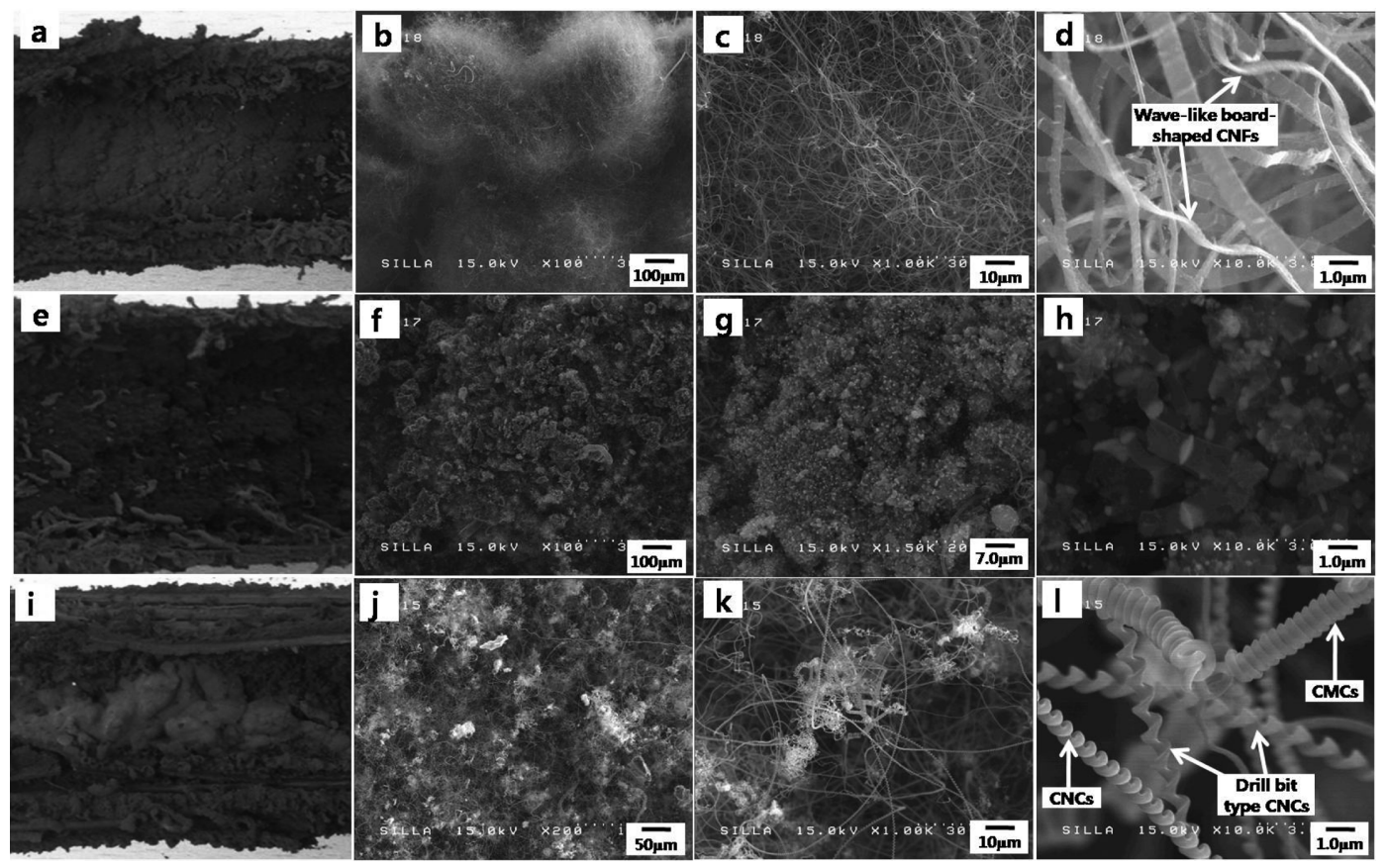

Fig. 3. Photographs and FESEM images according to the locations on the pretreated stainless steel substrate. (a) Photograph on the location A, (b) 100 magnified FESEM image on the location A, (c) 1,000 magnified FESEM image on the location A, (d) 10,000 magnified FESEM image on the location A, (e) Photograph on the location B, (f) 100 magnified FESEM image on the location B, (g) 1,500 magnified FESEM image on the location B, (h) 10,000 magnified FESEM image on the location B, (i) Photograph on the location C, (j) 200 magnified FESEM image on the location C, (k) 1,000 magnified FESEM image on the location C, and (1) 10,000 magnified FESEM image on the location $\mathrm{C}$. 


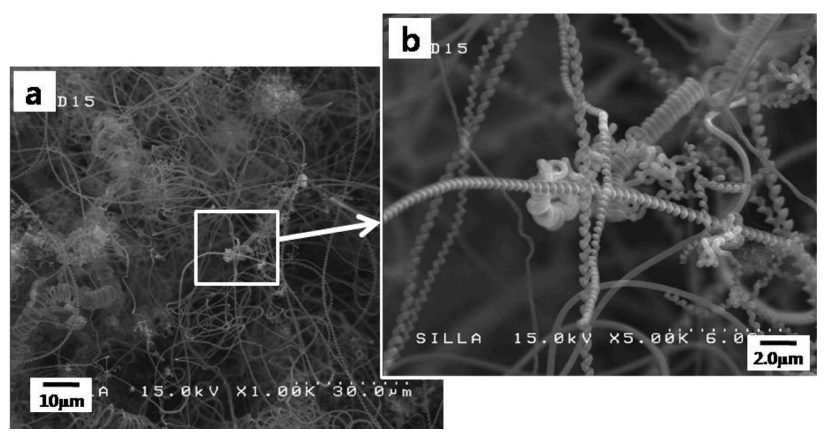

Fig. 4. (a) FESEM images for the as-grown carbon nanomaterials on the location $\mathrm{C}$ of the pretreated stainless steel substrate under the magnification 1,000 and (b) the 5,000 $\times$ magnified FESEM images for the square area of Fig. 4a.

location $\mathrm{C}$ seemed to reach almost $1 \mathrm{~mm}$. On the other hand, those of CMCs remained about several micrometers as shown in Fig. 4. These regular CMCs have constant coil pitch of $\sim 500 \mathrm{~nm}$ with little coil gap and have coil diameter of $\sim 2.2 \mu \mathrm{m}$. The carbon nanofilaments that built up the carbon coil have circular type shape.

The combined results of Figs. 1 4 give rise to the dominant formation of the carbon coils on the location $\mathrm{C}$ of the substrate, namely the grooves area. The cause for the formation of the carbon coils mostly on the location $\mathrm{C}$ of the substrate seems mainly due to the protru- sion induced by the grooves on the substrate surface. Namely, this protrusion on the substrate might provoke the sufficient surface energies for the interaction with the as-deposited carbon elements. Consequently they seemed to enhance more active sites for the initiation of the carbon coils. So, the location $\mathrm{C}$ of the substrate having the protrusion states would give the dominant formation of the carbon coils. Although the location B having a rough surface state may enhance the surface energies for the interaction between the substrate and the as-deposited carbon elements, the absolute magnitude seems to be lower than that of the location C. Therefore, the formation of the carbon coils seems to be dominant on the location $\mathrm{C}$, compared with the location B. Meanwhile, the carbon coils are rarely found on the pretreated stainless steel substrate without $\mathrm{Ni}$ catalyst particles, as the previous report [24].

To obtain the massive production yield of the carbon coils, we carried out the double pretreatment of the stainless steel substrate. Namely, we first pretreated the substrate by several circular-shaped grooves using a mechanical drill. Next, we pretreated again the same substrate by the unidirectional scratching using a $\mathrm{SiC}$ sand paper. As shown in Fig. 5, the formation of the carbon coils could be dramatically enhanced. Although

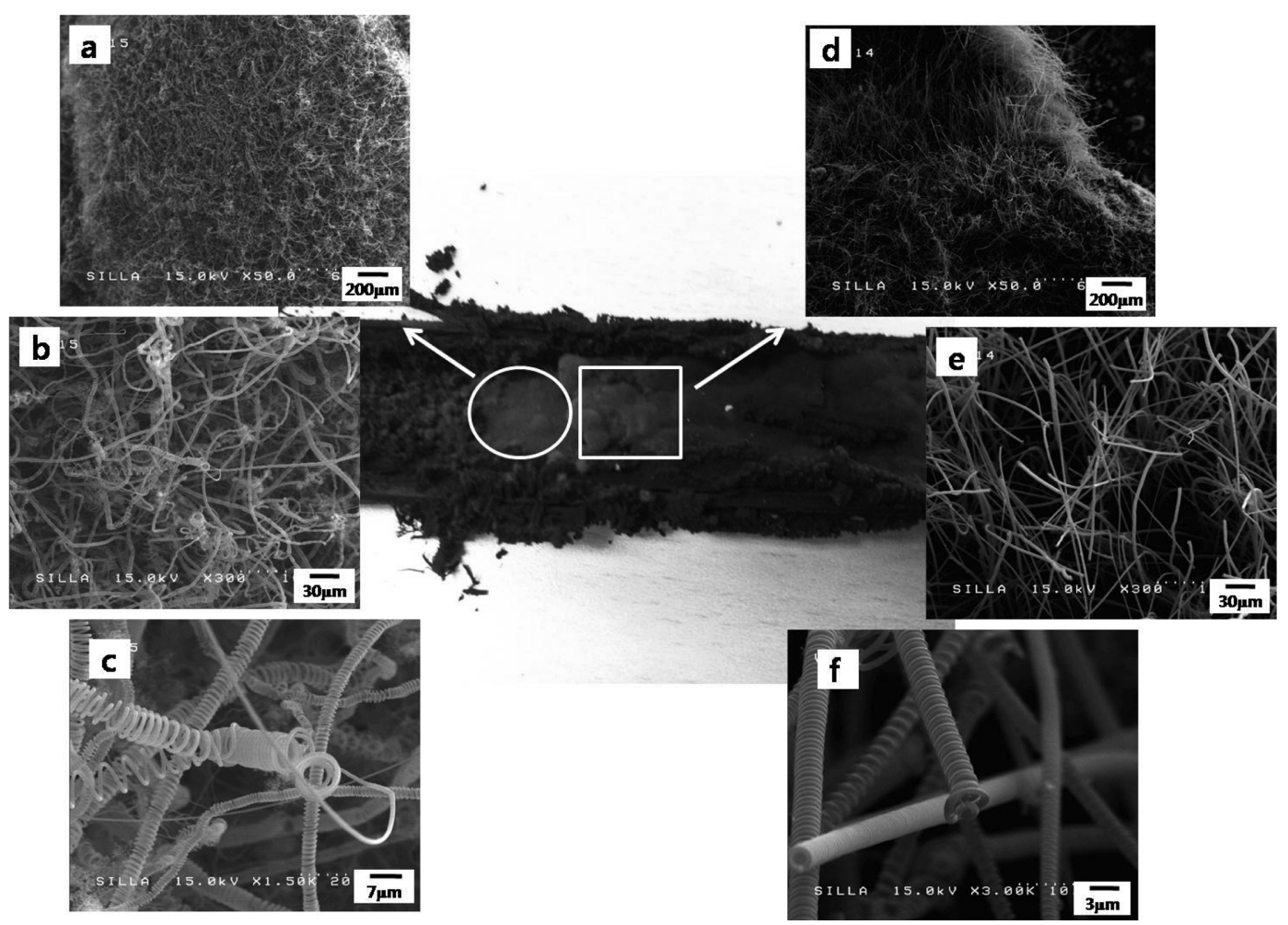

Fig. 5. Photograph of the as-grown carbon coils on the doubly pretreated (scratching + grooving) stainless steel substrate and FESEM images for the as-grown carbon coils of the circular area of the substrate under magnification (a) 50, (b) 300 , and (c) 1,500 and for the as-grown carbon coils of the square area of the substrate under magnification (a) 50, (b) 300, and (c) 3,000. 
the deposition aspect of the carbon nanomaterials seems to be different according to the locations on the substrate, the percentage of the carbon coils seems to increase up to nearly 35 percent, compared with the nearly 25 percent in the location $\mathrm{C}$ of the substrate. Noticeably, a massive amount of the well-developed carbon microcoils could be observed near the center area of the substrate (see the square are of the Fig. 5 and Figs. $5 d \sim$ f). These well-developed carbon microcoils have the linearshaped geometries. The coils diameters of the welldeveloped carbon microcoils were more or less reduced compared with those of the under-developed carbon microcoils (compare the diameters of the carbon microcoils in Fig. 5c with those of Fig. 5f). So, it is understood that the carbon microcoils are developed with reducing the diameters of the coils. The reason for this phenomenon seems to be due to the carbon coils formation mechanism as our previous report [26]. Namely, the precipitating rate of carbon elements according to the different crystal faces of the catalyst would be different. Therefore, the balance axis of the catalyst would be collapsed by the different growth rate of two carbon nanofilaments from the catalyst. Consequently, it gives rise to the spinning of the catalyst. The spinning rate increases with developing the carbon coils. As a result, the well-

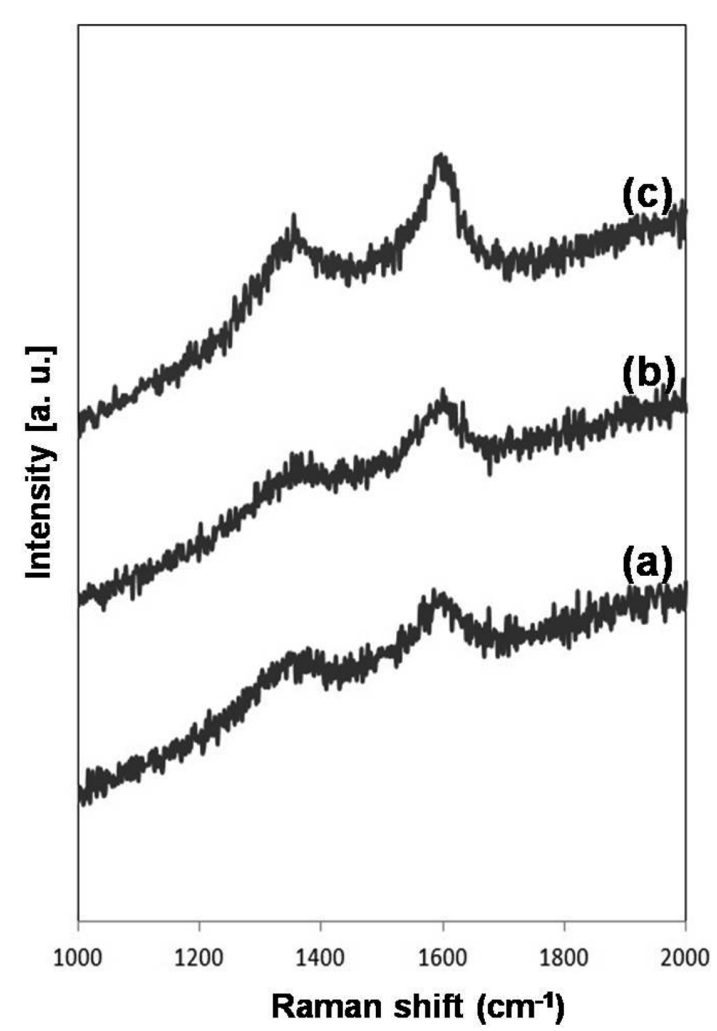

Fig. 6. Micro-Raman spectra of (a) location A, (b) location B, and (c) location $\mathrm{C}$ on the stainless steel substrate.
Table 2

The $\mathrm{I}_{\mathrm{D}} / \mathrm{I}_{\mathrm{G}}$ values calculated from the curve fitted Raman spectra

\begin{tabular}{ll}
\hline \hline Locations & $\mathrm{I}_{\mathrm{D}} / \mathrm{I}_{\mathrm{G}}$ \\
\hline $\mathrm{A}$ & 2.21 \\
$\mathrm{~B}$ & 2.35 \\
$\mathrm{C}$ & 1.92 \\
\hline
\end{tabular}

developed carbon coils have the compact and reduced coils diameters, compared with the under-developed carbon coils.

Meanwhile, the qualities of the carbon coils according to the locations on the substrate were also investigated by a micro-Raman spectrometer as shown in Fig. 6. The $D$ and $G$ peaks in all the different locations on the substrate were observed to be around $1350 \mathrm{~cm}^{-1}$ and $1580 \mathrm{~cm}^{-1}$, respectively. Table 2 shows $\mathrm{I}_{\mathrm{D}} / \mathrm{I}_{\mathrm{G}}$ values according to the locations on the substrate. $\mathrm{G}$ band is known to be related with the ordering of graphite layers within the material, while D-band is reported to be associated with the disordered states of $\mathrm{sp}^{2}$-hybridized carbon [27, 28]. As shown in Table $2, I_{D} / I_{G}$ value for the sample obtained in location $\mathrm{C}$ of the substrate, namely the sample dominated by the carbon coils, seems to be the lowest among the samples, indicating the largest presence of the ordered carbon phase in this sample. These tendencies indicate that the carbon coils for the samples of location $\mathrm{C}$, that is, in the case of the pretreated by several circular-shaped grooves using a mechanical drill, have the most polycrystalline development.

\section{Conclusions}

By several grooves formation on the stainless steel substrate, the large-scale synthesis of the carbon coils could be achieved. The protrusion induced by the grooves on the substrate surface was understood to be the main cause for the dominant formation of the carbon coils. Doubly pretreatment of the stainless steel substrate, namely scratching and grooving, could dramatically enhance the production yield of the carbon coils. Raman spectra confirm that the sample dominated by the carbon coils has the most polycrystalline development.

\section{References}

[1] L.J. Pan, T. Hayashida, M. Zhang and Y. Nakayama, "Field emission properties of carbon tubule nanocoils", Jpn. J. Appl. Phys. 40 (2001) L235.

[2] S. Amelinckx, X.B. Zhang, D. Bernaerts, X.F. Zhang, V. 
Ivanov and J.B. Nagy, "A formation mechanism for catalytically grown helix-shaped graphite nanotubes", Science 265 (1994) 635.

[3] S. Hokushin, L.J. Pan, Y. Konishi, H. Tanaka and Y. Nakayama, "Field emission properties and structural changes of a stand-alone carbon nanocoil", Jpn. J. Appl. Phys. 46 (2007) L565.

[4] S. Yang, X. Chen, S. Motojima and H. Iwanaga, "The Phenomenon of changing coiling-chirality in carbon nanocoils obtained by catalytic pyrolysis of acetylene with various catalysts", J. Nanosci. Nanotechnol. 4 (2007) 167.

[ 5 ] W.R. Davies, R.J. Slawson and G.R. Rigby, "An unusual form of carbon", Nature 171 (1953) 756.

[6 ] R.T.K. Baker, "Catalytic growth of carbon filaments", Carbon 27 (1989) 315.

[ 7 ] X. Wang, Z. Hu, Q. Wu, X. Chen and Y. Chen, "Synthesis of multi-walled carbon nanotubes by microwave plasma-enhanced chemical vapor deposition", Thin Solid Films 390 (2001) 130.

[ 8 ] A. Huczko, "Synthesis of aligned carbon nanotubes", Appl. Phys. A 74 (2002) 617.

[9] J.-H. Eum, S.-H. Kim, S.S. Yi and K. Jang, "Largescale synthesis of the controlled-geometry carbon coils by the manipulation of the $\mathrm{SF}_{6}$ gas flow injection time", J. Nanosci. Nanotechnol. 12 (2012) 4397.

[10] K. Akagi, R. Tamura and M. Tsukada, "Electronic structure of helically coiled cage of graphitic carbon", Phys. Rev. Lett. 74 (1995) 2307.

[11] V. Ivanov, J.B. Nagy, Ph. Lambin, A. Lucas, X.B. Zhang, X.F. Zhang, D. Bernaerts, G.V. Tendeloo, S. Amelinckx and J.V. Landuyt, "The study of carbon nanotubules produced by catalytic method", Chem. Phys. Lett. 223 (1994) 329.

[12] W. In-Hwang, H. Yanagida and S. Motojima, "Vapor growth of carbon micro-coils by the Ni catalyzed pyrolysis of acetylene using rotating substrate", Mater. Letters 43 (2000) 11.

[13] K. Hernadi, A. Fonseca, J.B. Nagy, D. Bernaerts and A.A. Lucas, "Fe-catalyzed carbon nanotube formation", Carbon 34 (1996) 1249.

[14] S. Motojima, Y. Itoh, S. Asakura and H. Iwanaga, "Preparation of micro-coiled carbon fibres by metal powder-activated pyrolysis of acetylene containing a small amount of sulphur compounds", J. Mater. Sci. 30 (1995) 5049.

[15] X. Chen and S. Motojima, "Morphologies of carbon micro-coils grown by chemical vapor deposition", J. Mater. Sci. 34 (1999) 5519.
[16] S. Motojima, S. Asakura, T. Kasemura, S. Takeuchi and H. Iwanaga, "Catalytic effects of metal carbides, oxides and $\mathrm{Ni}$ single crystal on the vapor growth of microcoiled carbon fibers", Carbon 34 (1996) 289.

[17] S. Yang, X. Chen and S. Motojima, "Tactile sensing properties of protein-like single-helix carbon microcoils", Carbon 44 (2006) 3352.

[18] K.-D. Kim, S.-H. Kim, N.S. Kim and D.-U. Kim, "Effect of the on/off cyclic modulation time ratio of $\mathrm{C}_{2} \mathrm{H}_{2} / \mathrm{H}_{2}$ flow on the low temperature deposition of carbon nanofilaments", J. Nanosci. Nanotechnol. 7 (2007) 3969.

[19] S.-H. Kim,"The geometry change of carbon nanofilaments by $\mathrm{SF}_{6}$ incorporation in a thermal chemical vapor deposition system", J. Korean Cryst. Growth Cryst. Technol. 21 (2011) 119.

[20] S.-H. Lee and S.-H. Kim, "Geometry variation for asgrown carbon coils under the minimized sulfur additive condition deposition system", J. Korean Cryst. Growth Cryst. Technol. 22 (2012) 213.

[21] S.-H. Lee and S.-H. Kim, "Effect of gas phase cycling modulation of $\mathrm{C}_{2} \mathrm{H}_{2} / \mathrm{SF}_{6}$ flows on the formation of carbon coils", J. Korean Vac. Soc. 18 (2012) 481.

[22] M. Asmann, J. Heberlein and E. Pfender, "A review of diamond CVD utilizing halogenated precursors", Diamond Relat. Mater. 8 (1999) 1.

[23] Y.-C. Jeon, J.-H. Eum, S.-H. Kim, J.-C. Park and S.I. Ahn, "Effect of the on/off cycling modulation time ratio of $\mathrm{C}_{2} \mathrm{H}_{2} / \mathrm{SF}_{6}$ flows on the formation of geometrically controlled carbon coils", Journal of Nanomaterials, vol. 2012, Article ID 908961.

[24] N.-K. Chang and S.-H. Chang, "High-yield synthesis of carbon nanocoils on stainless steel", Carbon 46 (2008) 1106.

[25] C.-C. Su and S.-H Chang, "Radial growth of carbon nanocoils on stainless wires coated with tin particles using chemical vapor despotion from acetylene", Materials Letters 65 (2011) 1114.

[26] S. Park, Y.C. Jeon and S.-H. Kim, "Effect of injection stage of $\mathrm{SF}_{6}$ flow on carbon micro coils formation", ECS J. Solid State Sci. and Technol. 2 (2013) M56.

[27] J. Robertson, "Diamond-like amorphous carbon," Mater. Sci. Eng. R 37 (2002) 129.

[28] E.P. Sajitha, V. Prasad, S.V. Subramanyam, S. Eto, K. Takai and T. Enoki, "Synthesis and characteristics of iron nano particles in a carbon matrix along with catalytic graphitization of amorphous carbon", Carbon 42 (2004) 2815. 